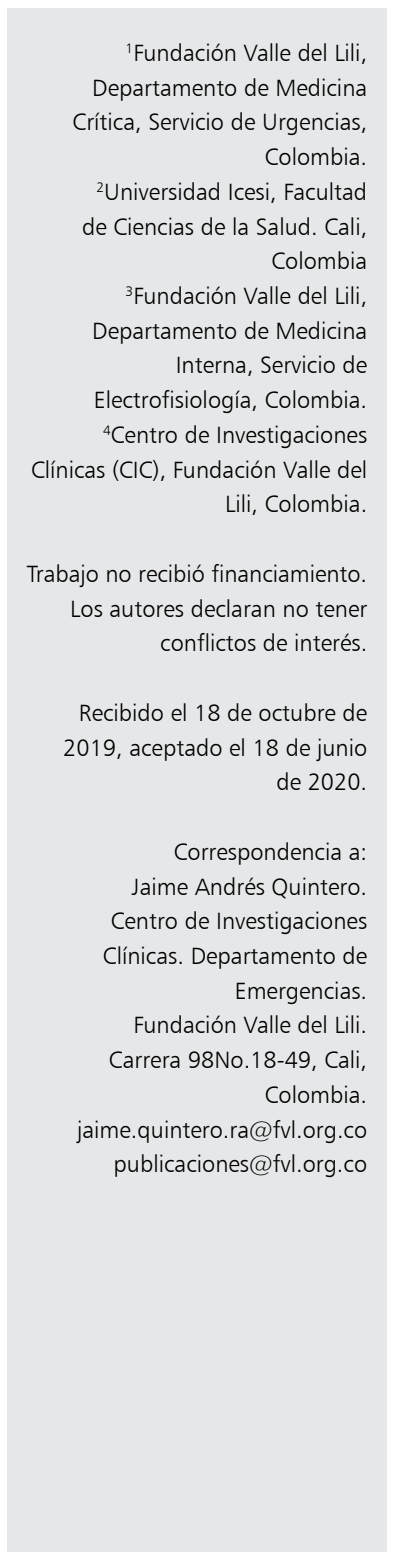

\section{Supervivencia a 30 días y 1 año de pacientes con muerte súbita con posterior colocación de dispositivo cardíaco implantable en un hospital de alta complejidad}

\author{
SANDRA CARVAJAL ${ }^{1}$, JAIME QUINTERO ${ }^{4}$, EDWARD OCAMPO ${ }^{1}$,
} PABLO PERAFÁN ${ }^{3}$, DANIEL CARVAJAL ${ }^{2}$, LUIS FERNANDO PAVA ${ }^{3}$

\section{Patients with sudden cardiac death receiving an implantable cardiac device: survival at 30 days and one year}

Background: The use of implantable cardiac devices in patients with sudden cardiac arrest has contributed to their survival. Aim: To determine the survival rate at 30 days and one year after hospital discharge of patients who had a cardiac arrest with subsequent placement of an implantable cardiac device. Material and Methods: Twenty-three patients older than 18 years who presented sudden extra-institutional or intra-institutional death with subsequent implantation of an implantable cardiac device and whose survival was recorded at 30 days and one year, were included. A univariate analysis was performed. Results: Eighteen patients had an extra institutional cardiac arrest. All patients were discharged alive. We could not ascertain the health status of one patient at follow-up. Twenty-one patients had a Cerebral Performance Category (CPC) of 1 at discharge. One patient died of a stroke within 30 days and one patient died due to an arrhythmic electrical storm one year later. Twenty patients survived at least one year after hospital discharge. Conclusions: Survival at 30 days and one year, was high in patients with sudden death or cardiac arrest who required intracardiac devices.

(Rev Med Chile 2020; 148: 772-777)

Key words: Cardiac Resynchronization Therapy Devices; Death, Sudden, Cardiac; Heart Arrest; Survival.
$\mathrm{E}$ n Colombia, la enfermedad cardiovascular se consolida como la primera causa de muerte en la población general entre el 2005 y 2017. Para el último año, las enfermedades isquémicas del corazón produjeron $53,3 \%$ (38.618) de las muertes dentro del grupo (Análisis de Situación de Salud en Colombia 2018). Dentro del espectro de presentaciones de la enfermedad cardiovascular la muerte súbita ha ido tomando mayor relevancia. Esta es definida, como la muerte inesperada que ocurre generalmente dentro de la primera hora del inicio de los síntomas en presencia de testigos y en el caso de no haber testigos se la considera cuando la muerte se produce 24 horas después que el individuo fue visto vivo en buenas condiciones generales $^{1,2}$. El paro cardíaco, se define como el cese de la actividad cardiaca lo cual compromete la perfusión de los órganos vitales del cuerpo. Es considerada según reporte de la Organización Mundial de la Salud (Global Health Estimates 
2016: Deaths by Cause, Age, Sex, by Country and by Region, 2000-2016. Geneva, World Health Organization; 2018.), como la causa primera de mortalidad en el mundo con 15,2 millones de muertes combinadas en 2016 convirtiéndose en un gran desafío para las sociedades actuales.

A pesar de las acciones preventivas como la modificación de estilos de vida, la utilización de medicación y procedimientos intervencionistas para disminuir la morbi-mortalidad, las patologías de origen cardiovascular siguen generando una alta carga en el sistema de salud y el impacto en la sobrevida de la población.

Junto a la innovación tecnológica, los procedimientos intervencionistas han alcanzado un logro importante en la generación de ayudas terapéuticas a dicha problemática. De ellas, la implantación de dispositivos cardíacos (marcapasos, cardiodesfibriladores implantables y cardioresincronizadores) han impactado en la sobrevida de pacientes, disminuyendo el alto riesgo de presentar paro cardíaco o muerte súbita cardíaca ${ }^{3,4}$. Se conoce que desde 1950 se utiliza la implantación de dispositivos cardíacos, con el fin de mejorar la condición y sobrevida de pacientes cardiópatas. La evidencia científica demuestra que la utilización de cardiodesfibriladores y cardioresincronizadores resulta en una mejoría significativa en la sobrevida ${ }^{5-7}$.

Sin embargo, en Colombia se desconoce el real impacto de la implementación de dichas medidas terapéuticas debido a las limitaciones de acceso a estas alternativas de manejo en el sistema de salud actual de nuestra región. Por otro lado, la falta de registro y seguimiento adecuado de dicha población, ha limitado la obtención de cifras objetivas que permitan arrojar datos precisos sobre el beneficio de su uso. Es por esto, que surge la necesidad de realizar una caracterización de la población que ha sido sometida a esta intervención y corroborar su estado vital a 1 mes y un año.

\section{Material y Método}

\section{Fuente de los datos}

El presente trabajo se diseñó como un estudio de revisión de casos retrospectivo derivada del registro CRECA (Sistema de registro de reanimación cardiopulmonar denominado Colombian Registry Cardiac Arrest CRECA-FVL). Este registro ins- titucional inició desde el 2011 y se ha enfocado en la recolección de datos de pacientes con activación del código azul en urgencias. Con esto se pretende acortar las brechas en el conocimiento de la secuencia de reanimación y las estrategias terapéuticas dentro del servicio de urgencias y en el manejo pos paro cardíaco, y así generar retroalimentación constante dentro del equipo.

La revisión de historias clínicas se realizó en el Hospital Universitario Fundación Valle del Lili (FVL) en el servicio de Urgencias el cual, cuenta con una capacidad para atender 103 pacientes al día distribuidos en las secciones de observación, hospitalización, reanimación y sillas. El servicio de Urgencias cuenta con un equipo y un sistema de triage, compuesto por jefes de enfermería con una preparación especial, Emergenciólogos y Médicos Internistas.

Para el proceso de revisión de Historias Clínicas, se extrajo la información del registro CRECA, el cual contiene la información de pacientes que ingresaron en código azul a la FVL desde el año 2011. Todos los datos recolectados son incluidos en una plataforma denominada BD clinic, administrada por el Centro de Investigaciones Clínicas de la FVL. El acceso está restringido al investigador principal, coinvestigadores y al epidemiólogo y estadístico. Los pacientes se tomaron de los registros de las historias clínicas digitales. La información fue suministrada al centro de Gestión de Información y Estadística de la FVL, solicitando los pacientes que cursaran con paro cardíaco e implantación de dispositivo intracardíaco durante el período de 2011 al 2018. Para los pacientes de este estudio, se filtraron solo los que cumplieron con los criterios de inclusión.

\section{Pacientes}

Los pacientes seleccionados cumplieron con los siguientes criterios de inclusión: ser mayores de 17 años con muerte súbita extra institucional e intra institucional entre 2011 al 2018 con posterior implantación de cardiodesfibrilador, cardioresincronizador o marcapasos. Se descartaron los pacientes con programación de implantación de dispositivo cardíaco como terapia de prevención primaria y prevención secundaria sin evento de paro cardíaco al ingreso a la institución.

Para el seguimiento de los pacientes al alta hospitalaria a los 30 días y al año, se revisaron las historias clínicas de control registradas en el 
sistema electrónico de historias clínicas. Además, se realizaron llamadas a los pacientes para determinar su estado al año del evento presentado.

\section{Análisis estadístico}

Se realizó un análisis descriptivo univariado. Las variables cualitativas se presentaron usando medidas de frecuencias relativas y absolutas. Las variables cuantitativas se expresarán a través de la medida de tendencia central y dispersión. Para este estudio, nuestro resultado de interés fue la supervivencia que se definió como vivo después de un paro cardíaco a los 30 días y al año. Esta variable se presentó en proporción al igual que las demás variables cualitativas.

El registro del cual se deriva este estudio fue aprobado por el comité de ética de investigación biomédica de la institución.

\section{Resultados}

En nuestra serie de casos, recolectados en un período de 8 años, se obtuvieron 23 casos de pacientes con muerte súbita y colocación de un dispositivo cardíaco implantable seguidos a 30 días y al año, para medir supervivencia por historia clínica. En 1 paciente no fue posible realizarle seguimiento, debido a que no fue posible contactarle con la información personal de dicha hospitalización. En todos los pacientes se pudo determinar la supervivencia a los 30 días y al año.

La mediana de edad poblacional fue de 66 años (RIC 53-70,5), con predominancia del sexo masculino $(74 \%)$. Todos los pacientes incluidos presentaron un CPC previo de 1. De los pacientes incluidos, 13 de ellos tenían antecedente de hipertensión arterial y 7 de ellos, antecedente de cardiopatía isquémica (Tabla 1).

De lo casos reportados, $18 / 23$ presentaron paro cardíaco extra institucional, siendo la fibrilación ventricular el principal ritmo de paro (5/18), seguido por la taquicardia ventricular $(3 / 18)$. En 7/18 no se pudo determinar el ritmo de paro por falta de información en la historia clínica. Los ritmos de paros intra institucionales (5/23) ocurrieron por fibrilación ventricular, actividad eléctrica sin pulso y asistolia. La asociación más frecuente de la muerte súbita fue la cardiopatía isquémica (14/23) (Tabla 2).

El estado neurológico al momento del alta mostró que 21/23 pacientes tenían un CPC con un puntaje de 1 . A 18 de los pacientes se les implantó un cardiodesfibrilador y a 5 restantes marcapasos.

Tres pacientes con desfibrilador recibieron descargas apropiadas por arritmias ventriculares antes de cumplido el año de seguimiento. Un paciente falleció antes de los 30 días por causa de un accidente cerebrovascular y 1 paciente antes del año por una tormenta eléctrica arrítmica. $\mathrm{Al}$ año sobrevivieron 20 pacientes (Tabla 3 ).

\section{Discusión}

Nuestro estudio fue realizado para describir el impacto en la sobrevida de pacientes con muerte súbita y la implementación de dispositivos cardíacos como medida terapéutica en dicha población a los 30 días y al año. El 95,7 logró sobrevivir a los 30 días y $91,3 \%$ al año del egreso hospitalario. El estado neurológico al momento del alta, en 91\%

Tabla 1. Descripción clínica de pacientes que presentaron muerte súbita con requerimiento de manejo con dispositivo implantable

\begin{tabular}{|lrr|}
\hline Datos demográficos & 66 & $(53-70,5)$ \\
\hline Edad** & & \\
Sexo, n (\%) & 6 & $(26,09)$ \\
Femenino & 17 & $(73,91)$ \\
Masculino & & \\
CPC antes del paro, n (\%) & 23 & $(100)$ \\
Puntuación 1 & & \\
Antecedentes, n (\%) & 13 & $(56,52)$ \\
HTA & 7 & $(30,43)$ \\
Cardiopatía isquémica & 4 & $(17,39)$ \\
DM & 4 & $(17,39)$ \\
Obesidad & 3 & $(13,04)$ \\
Tabaquismo & 1 & $(4,35)$ \\
Enfermedad arterial periférica & 1 & $(4,35)$ \\
IRC & & \\
Paro cerebro-cardio-respiratorio en los & 1 & $(4,35)$ \\
últimos 5 años & 4 & $(17,39)$ \\
Falla cardíaca & 3 & $(13,04)$ \\
Revascularización miocárdica & 2 & $(8,70)$ \\
Reemplazo valvular aórtico & & \\
** Mediana, RIC. & & \\
& &
\end{tabular}


Tabla 2. Caracterización del paro cardiaco

\begin{tabular}{|c|c|c|c|}
\hline Presentación del paro n (\%) & $\begin{array}{c}\text { Extra-institucional } \\
18(78,3)\end{array}$ & $\begin{array}{l}\text { Institucional } \\
5(21,7)\end{array}$ & $\begin{array}{c}\text { Total } \\
23(100)\end{array}$ \\
\hline \multicolumn{4}{|l|}{ Reanimación inmediata, n (\%) } \\
\hline Sí & $15(83,3)$ & $5(21,7)$ & $20(86,9)$ \\
\hline No & $3(16,7)$ & $0(0)$ & $3(13)$ \\
\hline \multicolumn{4}{|l|}{ Ritmo de paro, n (\%) } \\
\hline FV & $5(27,8)$ & $2(40)$ & $7(30,4)$ \\
\hline TV & $3(16,7)$ & $0(0)$ & $3(13)$ \\
\hline Asistolia & $1(5,6)$ & $1(20)$ & $2(8,7)$ \\
\hline AESP & $2(11)$ & $2(40)$ & $4(17,4)$ \\
\hline Sin dato & $7(38,9)$ & $0(0)$ & $7(30,4)$ \\
\hline \multicolumn{4}{|l|}{ Diagnóstico, n (\%) } \\
\hline Arritmia & $8(44,4)$ & $0(0)$ & $8(34,8)$ \\
\hline Coronariopatía & $9(50)$ & $5(100)$ & $14(60,9)$ \\
\hline Alteración morfología & $1(5,6)$ & $0(0)$ & $1(4,3)$ \\
\hline
\end{tabular}

Tabla 3. Desenlaces

\begin{tabular}{|c|c|}
\hline \multicolumn{2}{|l|}{ CPC a la salida del hospital, n (\%) } \\
\hline Puntuación 1 & $21(91,3)$ \\
\hline Puntuación 2 & $1(4,35)$ \\
\hline Puntuación 3 & $1(4,35)$ \\
\hline \multicolumn{2}{|c|}{ CPC a los 30 días de la salida del hospital, n (\%) } \\
\hline Puntuación 1 & $12(52,2)$ \\
\hline Puntuación 2 & $1(4,4)$ \\
\hline Puntuación 3 & $1(4,4)$ \\
\hline Sin dato & $9 \quad(39)$ \\
\hline \multicolumn{2}{|l|}{ Colocación de dispositivo intracardiaco, n (\%) } \\
\hline Desfibrilador cardioversor implantable & $18(78,3)$ \\
\hline Marcapasos & $5(21,7)$ \\
\hline \multicolumn{2}{|l|}{ Descargas a los 30 días, (\%) } \\
\hline Sin dato & $5(21,7)$ \\
\hline Ninguna & $17(73,9)$ \\
\hline Muerto & $1(4,4)$ \\
\hline \multicolumn{2}{|l|}{ Descargas durante el año, n (\%) } \\
\hline 1 o más descargas & $3(13)$ \\
\hline Sin dato & $4(17,4)$ \\
\hline Ninguna & $14(60,9)$ \\
\hline Muerto & $2(8,7)$ \\
\hline \multicolumn{2}{|c|}{ Estado a los 30 días de la salida del hospital, n (\%) } \\
\hline Vivos & $22(95,7)$ \\
\hline Muertos & $1(4,3)$ \\
\hline \multicolumn{2}{|l|}{ Estado al año de la salida del hospital, n (\%) } \\
\hline Vivos & $21(91,3)$ \\
\hline Muertos & $2(8,7)$ \\
\hline
\end{tabular}

de los pacientes fue CPC de 1, teniendo en cuenta que $9 \%$ restante presentó CPC de 2 y 3 .

Estos hallazgos son importantes, dado que a pesar de ser una muestra pequeña de pacientes, nos permite medir el impacto en sobrevida en una condición de alta mortalidad a nivel mundial. La terapia con los dispositivos cardíacos implantables ha demostrado su utilidad en la población de mayor riesgo de presentar arritmias ventriculares o de presentar muerte súbita (prevención primaria) y también en el contexto de pacientes con antecedentes de paro cardíaco/muerte súbita (prevención secundaria). Sin embargo, la utilización post paro cardíaco o muerte súbita cardiaca ha tomado relevancia como terapéutica a raíz de los hallazgos descritos en la literatura.

En un estudio realizado por Nagahara y colaboradores, incluyeron un total de 23 pacientes con paro cardíaco extra-institucional de origen cardíaco y 35 con paro intra-institucional en un período de 4 años, con implantación de algún tipo de dispositivo intracardíaco en la fase subaguda del evento, definieron resultados en supervivencia, estado neurológico a los 30 días del alta hospitalaria. Sus resultados muestran que $30 \%$ de los pacientes con paro extrainstitucional presentaron descargas terapéuticas de dicho dispositivo y ocurrió en la mitad de los pacientes con paro intrainstitucional. No se reportó ninguna muerte ${ }^{8}$. Estos resultados son congruentes con nuestros hallazgos respecto a la supervivencia. Suponemos que el resultado pri- 
mario es reflejo de la terapia con los dispositivos, puesto que el riesgo de sufrir nuevos eventos y una muerte prematura en estos pacientes es alta. La función neurológica no se alteró antes del evento (medida por CPC) en los pacientes que pudieron ser seguidos a los 30 días. Estos hallazgos también se correlacionan con nuestros resultados.

En 2003 B. Sterk y colaboradores realizaron un estudio donde se midió el uso de dispositivos cardíacos implantables en la práctica médica y adherencia a las guías. Se siguieron 70 pacientes con paro extra-hospitalario dados de alta de 15 hospitales, por más de un año. Solo 18 pacientes $(26 \%)$ tenían indicación de colocación de un desfibrilador automático, 13 pacientes (19\%) no tuvo un diagnóstico claro y no recibió implante y 37 pacientes $(53 \%)$ no tenían indicación y no lo recibieron. En $21 \%$ de los sobrevivientes se concluyó que los procedimientos diagnósticos fueron insuficientes referente a la indicación para la implantación de un desfibrilador o no se implantó aun cuando estaba indicado 9 . Esto demuestra la importancia de la adherencia a las guías de manejo, en específico los criterios de uso de los dispositivos cardíacos implantables. A pesar del pequeño tamaño de muestra, podemos observar una alta tasa de supervivencia de los pacientes que recibieron el dispositivo y en quienes se documentaron las indicaciones para recibirlo.

En 2012 Katritsis y col. publicaron una revisión crítica respecto a la evidencia existente de los casos de muerte súbita en el Reino Unido y el uso de cardiodesfibriladores implantables, planteando dichos eventos como fenómenos en crecimiento. Tras analizar los datos concluyen que para los pacientes con arritmias ventriculares sostenidas documentadas y/o paro cardíaco, los desfibriladores implantables confieren un beneficio de supervivencia. No se han establecido los beneficios de los DCI tanto en las personas mayores como en las mujeres o en las miocardiopatías no isquémicas. Su uso probablemente es más rentable solo cuando se usan en pacientes de alto riesgo, sin comorbilidades asociadas que limitan la esperanza de vida a 10 años $^{10}$. En ese orden de ideas, se muestra una concordancia con nuestros resultados, durante el seguimiento de los pacientes en este estudio.

Polsinelli y su grupo realizaron un estudio multicéntrico durante un período de 10 años, mostraron que la terapia con implantación de dispositivos cardíacos se asocia con un menor riesgo de muerte en pacientes con taquicardia ventricular, fibrilación ventricular y una tendencia no significativa en aquellos con asistolia ${ }^{11}$. A pesar de contar con una muestra poblacional mayor, su resultado respecto a la sobrevida demostró resultados similares con nuestra experiencia. Con respecto al ritmo de paro, ellos describieron que los pacientes que presentan con Fibrilación Ventricular tienen un riesgo menor de muerte que los que se presentan con Taquicardia Ventricular, Asistolia o Actividad Eléctrica sin Pulso, haciendo referencia a descripciones en otros estudios ${ }^{12,13}$. En nuestro estudio solo un paciente falleció por un evento arrítmico, con lo cual creemos que la utilización del desfibrilador si impactó en la supervivencia de nuestra población.

Moss y su equipo en el 2002 llevaron a cabo un estudio donde evaluaron el impacto de la utilización de DCI en pacientes con egreso después de un paro cardíaco. Con una muestra poblacional de 1.232 pacientes, concluyeron que la implantación de un DCI, puede estar asociada con una reducción de la mortalidad cardíaca después de ajustar otros factores de riesgo, y solo $40 \%$ de los pacientes que recibieron el DCI tuvieron una adecuada terapia ${ }^{14}$. Nuestro hospital es uno de los pocos en nuestra región, que cuenta con la infraestructura y personal para la realización de dicha intervención. Este es un punto relevante, puesto que la accesibilidad a dicha terapia está sesgada a un grupo poblacional reducido y sería apropiado reunir la población total de las instituciones para determinar las características y desenlaces generales.

\section{Limitaciones del estudio}

La principal limitación del estudio que hace difícil su aplicación es el tamaño limitado de la población disponible para el análisis de los resultados, siendo un estudio descriptivo retrospectivo sólo de la población atendida en la fundación Valle del Lili, la muestra poblacional se torna pequeña para hacer una extrapolación a nivel Municipal, departamental y Nacional.

Una de las mayores limitaciones para la realización de estudios que comparan la supervivencia de pacientes con dispositivos implantables y la población con manejo médico, recae en la poca utilización de estos dispositivos en la terapéutica, lo que limita el análisis en dichas poblaciones. En la ciudad, este tipo de terapia sólo puede ser 
ofrecida por pocos hospitales que cuentan con la infraestructura para realizarlo. Otro de los factores que influyen con el tamaño de la muestra, es la oportunidad con la que cuenta la población dado que no todos tienen el acceso a través de su afiliación al servicio de salud.

Evidenciamos un mejor resultado de supervivencia para la población con ritmo desfibrilable, y que se beneficia del implante de desfibrilador, lo cual arroja mejores resultados en esta subpoblación comparado con la población general, tanto en supervivencia como en buen desenlace neurológico.

Se sugiere realizar un estudio multicéntrico, lo cual, permitirá aumentar el grupo poblacional y analizar otros factores asociados como el tiempo que transcurre entre el retorno de la circulación espontánea hasta la colocación del implante.

Como conclusión principal, la supervivencia en la población con muerte súbita cardiaca e implante cardíaco en nuestro estudio fue alta.

\section{Referencias}

1. Regional Office for Europe - World Health Organization, Ischaemic Heart Disease Registers: Report on a Working Group Convened by the Regional Office for Europe of the World Health Organization, Copenhagen. 1969; 1: 1-23.

2. Björck G, Wikland B. "Sudden Death"-What Are We Talking About? Circulation 1972; 45: 256-8.

3. Connolly SJ, Hallstrom AP, Cappato R, Schron EB, Kuck KH, Zipes DP, et al. Meta-analysis of the implantable cardioverter defibrillator secondary prevention trials. Eur Heart J 2000; 21: 2071-8.

4. Bardy GH, Lee KL, Mark DB, Poole JE, Packer DL, Boineau R, et al. Sudden Cardiac Death in Heart Failure Trial (SCD-HeFT) Investigators. Amiodarone or an implantable cardioverter-defibrillator for congestive heart failure. N Engl J Med 2005; 352: 225-37.

5. McAnulty J, Halperin B, Kron J, Larsen G, Rait M, Swenson R, et al. A comparison of antiarrhythmic-drug therapy with implantable defibrillators in patients resuscitated from near-fatal ventricular arrhythmias. N Engl J Med 1997; 337 (22): 1576-83.

6. Kuck KH, Cappato R, Siebels J, Rüppel R. Randomized comparison of antiarrhythmic drug therapy with implantable defibrillators in patients resuscitated from cardiac arrest: The cardiac arrest study hamburg (CASH). Circulation 2000; 102 (7): 748-54.

7. Connolly SJ, Gent M, Roberts RS, Dorian P, Green MS, Klein GJ. Canadian Implantable Defibrillator Study (CIDS): Study design and organization. Am J Cardiol 1993; 72 (16): 1297-302.

8. Nagahara D, Hase M, Tsuchihashi K, Kokubu N, Sakurai S, Yoshioka T, et al. Long-term outcome of implanted cardioverter defibrillators in survivors of out-of-hospital cardiac arrest of cardiac origin. Circ J 2006; 70 (9): 1128-32.

9. Sterk B, van Alem AP, Tukkie R, Simmers TA, Koster RW. ICD-implantation guidelines versus clinical practice: a prospective study of out-of-hospital cardiac arrest survivors. Europace 2004; 6 (3): 179-83.

10. Katritsis DG, Josephson ME. Sudden cardiac death and implantable cardioverter defibrillators: Two modern epidemics? Europace 2012; 14 (6): 787-94.

11. Polsinelli VB, Wang NC, Kancharla K, Bhonsale A, Jain SK, Saba S. Implications of Initial Recorded Rhythm on Cardioverter-Defibrillator Insertion and Subsequent All-Cause Mortality in Sudden Cardiac Arrest Survivors. Am J Cardiol 2019; 124 (5): 709-14.

12. Madhavan M, Friedman PA, Lennon RJ, Prasad A, White RD, Sriram CS, et al. Implantable cardioverter-defibrillator therapy in patients with ventricular fibrillation out of hospital cardiac arrest secondary to acute coronary syndrome. J Am Heart Assoc 2015; 4 (2): 1-9.

13. Watanabe E, Tanabe T, Osaka M, Chishaki A, Takase B, Niwano S, et al. Sudden cardiac arrest recorded during Holter monitoring: prevalence, antecedent electrical events and outcomes. Heart Rhythm 2014; 11: 1418-25.

14. Moss AJ, Zareba W, Hall WJ, Klein H, Wilber DJ, Cannom DS, et al. Prophylactic implantation of a defibrillator in patients with myocardial infarction and reduced ejection fraction. N Engl J Med 2002; 346 (12): 877-83. 